\title{
Bioinformatic and biochemical analysis of the key binding sites of the pheromone binding protein of Cyrtotrachelus buqueti Guerin-Meneville (Coleoptera: Curculionidea)
}

\author{
Hua Yang ${ }^{\text {Equal first author, } 1}$, Yan-Lin Liu ${ }^{\text {Equal first author, } 1}{ }^{1}$, Yuan-Yuan Tao ${ }^{1}$, Wei Yang ${ }^{\text {Corresp., } 1}{ }^{1}$, Chun-Ping Yang ${ }^{1}$, Jing Zhang ${ }^{2}$, Li- \\ Zhi Qian ${ }^{1}$, Hao Liu ${ }^{1}$, Zhi-Yong Wang ${ }^{3}$ \\ ${ }^{1}$ Sichuan Agricultural University, Key Laboratory of Ecological Forestry Engineering of Sichuan Province/College of Forestry, Chengdu, Sichuan, China \\ 2 Provincial Key Laboratory of Agricultural Environmental Engineering, Sichuan Agricultural University, Chengdu, China \\ 3 Key Laboratory of Control and Resource Development of Bamboo Pest of Sichuan Province, Leshan, China \\ Corresponding Author: Wei Yang \\ Email address: ywei0218@aliyun.com
}

The bamboo snout beetle Cyrtotrachelus buqueti is a widely distributed wood-boring pest found in China, and its larvae cause significant economic losses because this beetle targets a wide range of host plants. A potential pest management measure of this beetle involves regulating olfactory chemoreceptors. In the process of olfactory recognition, pheromone-binding proteins (PBPs) play an important role. Homology modeling and molecular docking were conducted in this study for the interaction between CbuqPBP1 and dibutyl phthalate to better understand the relationship between PBP structures and their ligands. Site-directed mutagenesis and binding experiments were combined to identify the binding sites of CbuqPBP1 and to explore its ligand-binding mechanism. The 3D structural model of CbuqPBP1 has six a-helices. Five of these a-helices adopt an antiparallel arrangement to form an internal ligand-binding pocket. When docking dibutyl phthalate within the active site of CbuqPBP1, a $\mathrm{CH}-\pi$ interaction between the benzene ring of dibutyl phthalate and Phe69 was observed, and a weak hydrogen bond formed between the ester carbonyl oxygen and His53. Thus, Phe69 and His53 are predicted to be important residues of CbuqPBP1 involved in ligand recognition. Site-directed mutagenesis and fluorescence assays with a His53Ala CbuqPBP1 mutant showed no affinity toward ligands. Mutation of Phe69 only affected binding of CbuqPBP1 to cedar camphor. Thus, His53(Between $\alpha 2$ and a3) of CbuqPBP1 appears to be a key binding site residue, and Phe69(Located at $\alpha 3$ ) is a very important binding site for particular ligand interactions. 
1 Bioinformatic and biochemical analysis of the key binding sites of the 2 pheromone binding protein of Cyrtotrachelus buqueti Guerin-Meneville 3 (Coleoptera: Curculionidea)

4 Hua Yang ${ }^{1 \uparrow}$, Yan-Lin Liu ${ }^{1 \pi}$, Yuan-Yuan Tao ${ }^{1 \uparrow}$, Wei Yang ${ }^{1 *}$, Chun-Ping Yang ${ }^{1}$, Jing Zhang ${ }^{2}$, Li5 Zhi Qian ${ }^{1}$, Hao Liu ${ }^{1}$ and Zhi-Yong Wang ${ }^{3}$

$7 \quad{ }^{1}$ Key Laboratory of Ecological Forestry Engineering of Sichuan Province, College of Forestry, 8 Sichuan Agricultural University, Chengdu, Sichuan 611130, China.

$9 \quad{ }^{2}$ Provincial Key Laboratory of Agricultural Environmental Engineering, Sichuan Agricultural

10 University, Chengdu, Sichuan 611130, China.

$11{ }^{3}$ Key Laboratory of Control and Resource Development of Bamboo Pest of Sichuan Province,

12 Leshan, Sichuan, 614000, China.

$14{ }^{*}$ Corresponding Author:

15 Wei Yang

16 E-mail: ywei0218@aliyun.com

17 TThese authors contributed equally to this article 


\section{Abstract}

20 The bamboo snout beetle Cyrtotrachelus buqueti is a widely distributed wood-boring pest found

21 in China, and its larvae cause significant economic losses because this beetle targets a wide range

22 of host plants. A potential pest management measure of this beetle involves regulating olfactory

23 chemoreceptors. In the process of olfactory recognition, pheromone-binding proteins (PBPs)

24 play an important role. Homology modeling and molecular docking were conducted in this study

25 for the interaction between CbuqPBP1 and dibutyl phthalate to better understand the relationship

26 between PBP structures and their ligands. Site-directed mutagenesis and binding experiments

27 were combined to identify the binding sites of CbuqPBP1 and to explore its ligand-binding

28 mechanism. The 3D structural model of CbuqPBP1 has six $\alpha$-helices. Five of these $\alpha$-helices

29 adopt an antiparallel arrangement to form an internal ligand-binding pocket. When docking

30 dibutyl phthalate within the active site of CbuqPBP1, a $\mathrm{CH}-\pi$ interaction between the benzene

31 ring of dibutyl phthalate and Phe69 was observed, and a weak hydrogen bond formed between

32 the ester carbonyl oxygen and His53. Thus, Phe69 and His53 are predicted to be important

33 residues of CbuqPBP1 involved in ligand recognition. Site-directed mutagenesis and

34 fluorescence assays with a His53Ala CbuqPBP1 mutant showed no affinity toward ligands.

35 Mutation of Phe69 only affected binding of CbuqPBP1 to cedar camphor. Thus, His53(Between

$36 \alpha 2$ and $\alpha 3$ ) of CbuqPBP1 appears to be a key binding site residue, and Phe69(Located at $\alpha 3$ ) is a

37 very important binding site for particular ligand interactions.

38 Keywords: Cyrtotrachelus buqueti, pheromone binding protein, bioinformatics, site-directed 
39 mutagenesis, fluorescence assay

40

Peer] reviewing PDF | (2019:04:36358:3:0:NEW 19 Aug 2019) 


\section{Introduction}

42 During long-term evolution insects have developed a sensitive sense of smell, which enables

43 insects to detect external volatile semiochemicals when searching for various environmental cues,

44 such as foraging for food, finding a breeding partner and locating a spawning ground(Gu et al.,

45 2011; Larsson et al., 2004). Tentacles are the main olfactory part of insects and contain a large

46 variety of receptors. Receptors are widely distributed with various olfactory-rated functional

47 proteins, including odorant binding proteins (OBPs), chemosensory proteins (CSPs) and

48 olfactory receptors (Ors). OBPs are divided into pheromone binding proteins (PBPs), general

49 odorant binding proteins (GOBPs) and antennal binding proteins (ABPs)(Vogt and Riddiford

50 1981). Research on the binding mechanism between OBPs and ligand molecules has been a

51 major focus of research, including defining the three-dimensional (3D) structure of these OBPs.

52 Kruse et al., (2003) and Thode et al., (2008) initially analyzed the general odorant binding

53 protein (LUSH) of Drosophila melanogaster and the crystal structure of the complex between

54 LUSH and alcohol, and clarified that Thr57 is a key residue involved in ligand interaction. In

55 accordance with X-ray diffraction analysis of the pheromone binding protein BmorPBP of

56 Bombyx mori and structure of bombykol, Sandler et al. (2000) discovered that Ser56 of this

57 protein played a key role by forming a hydrogen bond with the ligand bombykol. According to

58 the structures of odorant binding protein CquiOBP1 and MOP of Culex quinquefasciatus, Mao et

59 al. (2010) discovered that instead of hydrogen bonds, the interaction between protein and ligand

60 was driven by van der Waals forces and hydrophobic interactions. Based on the structure 
61 between the odorant binding protein HoblOBP2 of Holotrichia oblita and ethyl

62 benzenecarboxylate, Zhuang et al. (2013) discovered that this protein-ligand complex involved

63 both van der Waals forces and hydrophobic interactions. Currently, high-resolution structural

64 data describing the complex between the pheromone binding protein of Cyrtotrachelus buqueti

65 and an odor molecule is unavailable, and thus information about the mode of action of this

66 protein remains unresolved.

67 Cyrtotrachelus buqueti (C. buqueti) also named as the bamboo snout beetle, belongs to

68 Cyrtotrachelus, Curculionidea, Coleoptera. C. buqueti is endangering survival of bamboo shoots

69 from 28 different types of bamboos, including Bambusa, Dendrocalamopsis and Dendrocalamus.

70 In particular, the larvae prefer the bamboo shoots of Phyllostachys pubescens,

71 Dendrocalamopsis oldhami, Bambusa textilis, Bambusa pervariabilis, Dendrocalamopsis daii

72 and other sympodial bamboo species(Ju et al., 2005; Wang et al., 2005). C. buqueti is distributed

73 widely in the Sichuan Province, Chongqing City, Guangdong Province, Guangxi Province,

74 Guizhou Province and other provinces (districts) as well as Vietnam, Burma, Thailand and other

75 countries and regions in Southeast Asia(Yang et al., 2009). C. buqueti is one of 233 hazardous

76 forest pests issued in 2003 for the first time(Yang et al., 2015).

77 Currently, research on $C$. buqueti has mainly concentrated on a description of the general

78 biological characteristics and common chemical pest control approaches(Ju et al., 2005; Wang et

79 al., 2005; Yang et al., 2010; Yang et al., 2009). The development of sex attractants remains

80 poorly understood. Mang et al. (2012) have extracted and studied the body surface 
81 semiochemicals of $C$. buqueti adults, whereas Yang et al. (2017a) have constructed a

82 transcriptome library of $C$. buqueti and analyzed the sex pheromone binding protein gene. Yang

83 et al. (2017b) have also cloned the sex pheromone binding protein gene that codes for the protein

84 CbuqPBP1, and conducted fluorescence competitive binding assays for many types of simple

85 odor substances. Based on a phylogenetic analysis (Yang et al., 2018; summarized in

86 supplementary information) CbuqPBP1 was quite similar to the PBPs of other insects. Amino

87 acid sequence similarity analysis showed that CbuqPBP1 had $37.68 \%$ similarity with 27 PBPs of

8817 insects of Coleoptera and Lepidoptera. The similarities with PBPs from Coleoptera and

89 Lepidoptera were $38.47 \%$ and $52.39 \%$ respectively.

90 In this paper, homology modeling of the pheromone binding protein CbuqPBP1 of $C$.

91 buqueti has been conducted to create a 3D model of the protein. Molecular docking has also been

92 carried out to define the interaction mode between the ligand dibutyl phthalate and CbuqPBP1.

93 Two key binding site residues, Phe69 and His53, were identified from this modeling and were

94 mutated. Fluorescence competitive binding assays were conducted for these mutants and binding

95 mechanism between CbuqPBP1 and odor molecules was analyzed. The results provide a

96 platform for using pheromones to prevent and control C. buqueti efficiently.

\section{Materials}


100 Three compounds were chosen to investigate the ligand-binding specificity of CbuqPBP1.

101 Ligands of the highest purity were purchased from Aladdin (Shanghai, China) and stored in 102 accordance with the manufacture's specifications. The sequence of CbuqPBP1 was taken from 103 the GenBank with accession number KU845733.1.

\section{Alignment and homology modeling}

105 The amino acid sequence of CbuqPBP1 was downloaded from the GenBank and Blast was used 106 to search against the CbuqPBP1 protein sequence in the Protein Data Bank to identify a 107 structural template. Software Modeller 9.19 (http://salilab.org/modeller/) was used for homology 108 modeling based on the sequence comparison results with the structural template sequence 109 identified. The 3D structure obtained from modeling was evaluated with SAVES v5.0 110 (https://servicesn.mbi.ucla.edu/SAVES/). After confirming the models, the Chiron 111 (http://redshift.med.unc.edu/chiron/login.php) on-line server was used for optimization. Modeller

1129.19 was used to optimize loop regions and PyMOL was used to analyze structural 113 characteristics and to search for ligand binding sites.

\section{Molecular docking}

Based on the established homology model, the docking program AUTODOCK vina 1.1.2 was used to find the potential binding mode between CbuqPBP1 and the ligand dibutyl phthalate. Dibutyl phthalate with strong affinity is a female pheromone of the giant bamboo weevil, which plays a role in the process of male individual searching for female individual. ChemBioDraw 
119 Ultra 14.0 was used to simulate the structure of dibutyl phthalate and to generate a 3D structure 120 of the ligand. Energy optimization was conducted using the MMFF94 force field and Autodock 121 Tools 1.5.6 was used to create the PDBQT format(Huey et al., 2007; Morris et al., 2009). 122 Binding coordinates of CbuqPBP1 and dibutyl phthalate were set to: center_x $=22.389$, center_y $123=-25.143$, center $\_z=1.08$, and size $\_x=15$, size $\_y=15$, size $\_z=15$. Parameter exhaustiveness 124 was set to 20 and default values were used for other parameters to increase the calculation 125 accuracy. Finally, the conformation with the highest score was selected and PyMoL 1.7.6 was 126 used for visual inspection and analysis of the structural data.

\section{Site-directed mutagenesis}

128 The CbuqPBP1 coding sequence was mutated to yield the two mutants CbuqPBP1-Phe69A 129 (phenylalanine to alanine at position 69) and CbuqPBP1-His53A (histidine to alanine at position 130 53). PCR reactions were used to form overlapping chains. The extension of overlapping chains 131 was used to splice segments in a superimposed manner. Primer5 was used to design primers 132 (Table 1). Three rounds of PCR amplification were conducted after designing primers. 133 Expression vectors (pET-28a(+)/PBP1-Phe69A, pET-28a(+)/PBP1-His53A and pET$13428 \mathrm{a}(+) / \mathrm{PBP} 1)$ were generated and transformed into Escherichia coli BL21(DE3) competent cells 135 for protein overexpression. Recombinant proteins produced were detected by SDS-PAGE 136 analysis.

\section{Expression and purification of the native protein and mutants}


138 Expression plasmids were transformed into E. coli TOP10 competent cell and plated on agar 139 plates. Several colonies were selected randomly for overnight cultivation in LB media and 140 plasmids were extracted for sequencing. Mutant plasmids pET-28a(+)/PBP1- Phe69A and pET$14128 \mathrm{a}(+) / \mathrm{PBP} 1-$ His53A with the correct sequence were transformed into E. coli BL21(DE3) competent cells, and cells were grown to an optical density at $600 \mathrm{~nm}\left(\mathrm{OD}_{600}\right)$ of 0.6 . IPTG was added to the culture to a final concentration of $1 \mathrm{mM}$ and cells were further grown at $37{ }^{\circ} \mathrm{C}$ with 144 shaking for $3 \mathrm{~h}$ to induce protein expression(Deng et al., 2011). After harvesting cells by centrifugation, ultrasound sonication was used to disrupt cells $(200 \mathrm{~W}, 3 / 4 \mathrm{~s}, 25-30 \mathrm{~min})$. The supernatants and sediments were collected under low temperature centrifugation (16000 g-force, $50 \mathrm{~min}$ ) and SDS-PAGE detection was conducted. Nickel affinity (Ni-NTA) was used to purify recombination proteins, and the purified proteins were stored in Tris- $\mathrm{HCl}$ buffer $(\mathrm{pH} 7.4,50$ $\mathrm{mM})$. To avoid the function of the protein being affected by the His-tag, recombinant bovine enterokinase was used to remove the His-tag and the protein was re-purified and collected. Purity was confirmed by SDS-PAGE analysis.

\section{Fluorescence assay}

153 To measure the affinity of the fluorescent ligand N-phenyl-1-naphthylamine (1-NPN) toward 154 CbuqPBP1, a $2 \mu \mathrm{M}$ solution of protein in $50 \mathrm{mM}$ Tris- $\mathrm{HCl}$, $\mathrm{pH} 7.4$, was titrated with aliquots of $1 \mathrm{mM} 1-\mathrm{NPN}$ dissolved in methanol to a final concentration of $16 \mu \mathrm{M}$. The probe was excited at $337 \mathrm{~nm}$ and emission spectra were recorded between 350 and $550 \mathrm{~nm}$. To evaluate the effect of $\mathrm{pH}$ on the binding affinity of CbuqPBP1, we also measured its binding with 1-NPN over a $\mathrm{pH}$ 
158

159

160

161

162

163

164

165

166

167

168

169

170

171

172

173

174

175

176

range of 4.5-9.0. The displacement of 1-NPN by selected ligands was measured in a competitive binding assay using both the protein and 1-NPN at $2 \mu \mathrm{M}$. The mixtures were titrated with $1 \mathrm{mM}$ methanol solutions of each competitor at concentrations of $2-16 \mu \mathrm{M}$. The fluorescence of the mixture was recorded after $5 \mathrm{~min}$. Dissociation constants for 1-NPN and the stoichiometry of binding were obtained from Scatchard plots of the binding data using the Prism software. For other competitor ligands, the dissociation constants were calculated from the corresponding half maximal inhibitory concentration $\left(\mathrm{IC}_{50}\right)$ values using the equation: inhibitory constant $\mathrm{Ki}=$ $\left[\mathrm{IC}_{50}\right] /\left(1+[1-\mathrm{NPN}] / \mathrm{K}_{1-\mathrm{NPN}}\right)$, where $[1-\mathrm{NPN}]$ is the free concentration of $1-\mathrm{NPN}$ and $\mathrm{K}_{1-\mathrm{NPN}}$ is the dissociation constant of the protein/1-NPN complex.

\section{Results}

\section{Three-dimensional model of CbuqPBP1}

On the basis of the Blast search against the Protein Data Bank, two types of insect odor proteins with known structures and quite similar sequences to the CbuqPBP1 sequence were found. These two odorant binding proteins were Nasonovia ribisnigri OBP3 (NribOBP3 PDB ID: 4Z45_A) and Megoura viciae OBP3 (MvicOBP3 PDB ID: 4Z39_A). The total sequence identity between the target (CbuqPBP1) and the template protein (NribOBP3) is 33\% (Cavasotto and Phatak 2009)(Fig. 1A). The resolution of the template is $2.02 \AA$. 
177 to the 3D structure of the template NribOBP3 (Fig. 1C). The structural characteristics of 178 CbuqPBP1 are similar to other sex pheromone binding proteins and include six $\alpha$-helices: 179 residues 26-36 ( $\alpha 1), 44-51(\alpha 2), 59-72(\alpha 3), 83-94(\alpha 4), 101-114(\alpha 5)$ and 123-137 ( $\alpha 6)$. Six

180

181

182 183 184

conserved cysteine residues stabilize the protein structure by forming three disulfide bonds.

Disulfide bond Cys36-Cys67 connects $\alpha 1$ and $\alpha 3$, Cys63-Cys121 connects $\alpha 3$ and $\alpha 6$, and Cys110-Cys130 connects $\alpha 5$ and $\alpha 6$. Five of the $\alpha$-helices adopt an antiparallel arrangement $(\alpha 1, \alpha 3, \alpha 4, \alpha 5$ and $\alpha 6)$ and form an internal binding pocket. $\alpha 2$ forms a cover-type structure or lid above the pocket, which stabilizes this structure.

The result of further rationality estimates by Pro-CHECK (Fig. 1D) was that $88.4 \%$ residues were in the favored regions (red area A, B and L), 10.1\% of the residues fall into additionally allowed regions (bright yellow area $\mathrm{a}, \mathrm{b}, 1, \mathrm{p}$ ) and $0.8 \%$ residues have backbone torsion angles that fall into generously allowed regions (light yellow area $\sim a, \sim b, \sim 1, \sim p$ ). The percentage sum of residues in the allowed regions was $99.3 \%$, which was higher than $95 \%$. This result showed that the constructed 3D structure of CbuqPBP1 was a high-quality model.

Energy assessment was performed on ProSa (Fig. 1E). The shadow part is Z-score value of all proteins similar to Cbuq PBP1 protein in PDB database, and the black spot is Z-score value of Cbuq PBP1 protein, which is -4.35 . The Z-score value of template protein NOBP3 is -5.87 in the range of Z-score of known reasonable structural proteins, which indicates that the modeling structure is more stable than template structure. This indicates that the homologous modeling Institute is more stable than template structure. The constructed CBuq PBP1 protein is 
197 198

200

201

202

203

204

205

206

207

208

209

210

211

212

213

214

215

reasonable in energy.

\section{Molecular docking}

To research characteristics of CbuqPBP1 binding with odor molecules, dibutyl phthalate (Fig. 2A), which interacts with CbuqPBP1 favorably, was selected to construct a complex between CbuqPBP1 3D model and dibutyl phthalate. Such a model should clarify the mode of interaction of dibutyl phthalate with CbuqPBP1 at the molecular level. We have docked dibutyl phthalate with the active pocket of CbuqPBP1, with a binding energy of $-6.4 \mathrm{kcal} / \mathrm{mol}$. Generally, compound dibutyl phthalate bound to the active pocket of CbuqPBP1 with a compact conformation (Fig. 2B).

The benzene ring and one aliphatic chain of dibutyl phthalate were located in the hydrophobic region at the bottom of the pocket. Strong hydrophobic interactions formed between the ligand and residues Leu3, Leu4, Leu5, Leu29, Leu50, Pro56, Ile65 and Phe69. Another side chain of dibutyl phthalate was located at the opening of the pocket. Based on detailed analysis, a $\mathrm{CH}-\pi$ interaction may occur between the benzene ring of dibutyl phthalate and residue Phe69. Moreover, an important long-range hydrogen bond (3.3 $\AA$ ) can form between one ester carbonyl oxygen of dibutyl phthalate and residue His53 (Fig. 2C). All aforementioned interactions enable the formation of a stable complex between dibutyl phthalate and CbuqPBP1.

\section{Site-directed mutagenesis of CbuqPBP1 and binding specificities of mutants}

After double enzyme digestion with restriction enzymes $N d e$ I and Xha I, mutant plasmids pET- 
$21628 \mathrm{a}(+) /$ CbuqPBP1-His53A and pET-28a(+)/CbuqPBP1-Phe69A, and the original plasmid pET-

$21728 \mathrm{a}(+) / \mathrm{PBP} 1$ formed bands in an agarose gel that were $400 \mathrm{bp}$ in length (Fig. 3). After SDS-

218 PAGE analysis of protein overexpression, three specific bands with molecular weights of $16 \mathrm{kDa}$

219 were observed in the SDS-PAGE gel, which is consistent with expected molecular weight of the

220 target proteins (Fig. 4).

After ultrasonication to disrupt the bacteria and release the recombinant proteins (including

222 His tag), SDS-PAGE analysis could be conducted (Fig. 5). All recombinant proteins were found

223 in the supernatant part of the disrupted cells. After purification, recombinant bovine enterokinase

224 was used to cleave the His-tag and following a further round of purification pure recombinant

225 protein samples were obtained.

1-NPN was selected as the fluorescent probe. Fluorescence competitive binding assays were

Phe69A proteins. The fluorescence peak maximum in the presence of the recombinant proteins

was recorded at different concentrations. The Scatchard equation was used to calculate the 
236 fluorescence binding assay test(Yang et al., 2017b). Fluorescence competitive binding assays

237 were conducted with CbuqPBP1, CbuqPBP1-His53A and CbuqPBP1-Phe69A (Fig. 7). Based on

238 the results, CbuqPBP1 bound favorably with dibutyl phthalate, benzothiazole and cedar camphor.

239 The binding ability of CbuqPBP1-His53A with the three types of odor substances was essentially

240 lost. The binding ability of CbuqPBP1-Phe69A mutant with cedar camphor was significantly

241 reduced, whereas affinity toward the other two odor substances was not significantly different

242 from that of the wild-type protein (Table 2).

Discussion

245 Currently, 3D structure prediction of odorant binding proteins through homology modeling has

246 been conducted for proteins from Choristoneura rosaceana, Choristoneura murinana,

247 Pectinophora gossypiella, Heliothis assulta, Spodoptera exigua, Spodoptera exigua, Holotrichia

248 oblita, lettuce Aphidoidea, Megoura viciae and other insects(Northey et al., 2016; Sun et al.,

249 2013; Wang et al., 2015). On the basis of homology modeling of pheromone binding protein

250 CbuqPBP1 of $C$. buqueti, the 3D structure is composed of six $\alpha$-helices, which packed together

251 and were stabilized by three disulfide bonds. Disulfide bonds Cys36-Cys67, Cys63-Cys121 and

252 Cys110-Cys130 connected $\alpha 1$ and $\alpha 3, \alpha 3$ and $\alpha 6, \alpha 5$ and $\alpha 6$ respectively. Five of the $\alpha$-helices

253 arranged in an antiparallel manner to form an internal binding pocket(Tian et al., 2017). $\alpha 2$

254 formed a cover-type structure above the pocket, which was similar to Holotrichia oblita

255 HoblOBP2(Zhuang et al., 2013) structures. As for 3D structure of Bombyx mori BmorPBP, four 
256 antiparallel $\alpha$-helices formed a hydrophobic pocket and $\alpha 2$ and $\alpha 3$ did not participate in the

257 formation of the pocket(Sandler et al., 2000). This might be due to differences in hydrophobic

258 pocket of the 3D structure of odorant binding proteins from different insects. Such differences

259 are likely to be closely related to the function of these proteins.

260 According to research, odorant binding proteins from some insects interact with their cognate

261 ligand through hydrogen bonds and hydrophobic interactions, whereas other odorant binding

262 proteins from other insects interact with odorants via van der Waals forces and hydrophobic

263 interactions(Sandler et al., 2000). In this report, a CH- $\pi$ interaction formed between the benzene

264 ring of dibutyl phthalate and Phe69. This $\mathrm{CH}-\pi$ interaction is generally considered to be a

265 relatively weak hydrogen bond. Previous research has indicated that $\mathrm{CH}-\pi$ interactions are

266 important in carbohydrate-protein identification processes, where the $\mathrm{CH}-\pi$ features as a

267 synergistic interaction that plays an important role in stabilizing the structure of the complex

268 (Jiang et al., 2009; Kozmon et al., 2011). The CH- $\pi$ interaction involves a nonpolar interaction

269 between the $\mathrm{CH}$ proton and electron-rich aromatic ring $\pi$ electron cloud system, playing a similar

270 role to hydrogen bonding in controlling crystal stacking, maintaining biomolecular structures and

271 participating in molecular recognition processes(Ye et al., 2015; Zhao et al., 2014). Therefore,

272 we hypothesize that the $\mathrm{CH}-\pi$ interaction may play a role in binding and stabilizing the

273 interaction with odor molecules.

274 An ester carbonyl oxygen from dibutyl phthalate and His53 from the protein formed a weak

$2753.3 \AA$ hydrogen bond. Such a hydrogen bond has been reported in odorant binding proteins of 
276 other insects, for example, BmorPBP1 of $B$. mori and pheromone compound interacted through a

277 hydrogen bond. General odorant binding protein (LUSH) from Drosophila melanogaster and the

278 pheromone binding protein (ApolPBPl) from Antheraea polyphemus interact with their cognate

279 ligands through hydrogen bonds(Damberger et al., 2007; Thode et al., 2008).

According to the fluorescence competitive binding assay, mutant $\mathrm{pET}-28 \mathrm{a}(+) / \mathrm{PBP} 1-\mathrm{His} 53 \mathrm{~A}$ could not interact with odor substances. Replacing His53 with alanine removed the ability of the mutant to form this key hydrogen bond with ligands, and therefore the ability to bind with odor substances. Thus, His53 is a key binding site residue of the pheromone binding protein of $C$. buqueti. Mutein pET-28a(+)/PBP1-Phe69A did not bind cedar camphor. However, only a decrease in binding ability toward dibutyl phthalate and benzothiazole was observed. These observations indicate that only a small number of intermolecular forces between the protein and odor molecules were affected by this mutation(Zhuang et al., 2014). Thus, the binding affinity had been reduced, but not completely lost.

\section{Conclusions}

In summary, we hypothesized that the CbuqPBP1 interaction and release of the ligand involves

hydrogen bond formation via His53. Phe69 is the binding site for CbuqPBP1 to combine with odor substance; however, Phe69 is not a key binding site residue. Moreover, these observations showed that the combination between CbuqPBP1 and ligands was affected by loss of hydrogen bonding and other intermolecular forces, and the interaction between CbuqPBP1 and ligands involves the joint action of many acting forces and the binding site(Li et al. 2016). 
296 Acknowledgments

297 This work was funded by the key fund of the education department in Sichuan (17ZB0344) and 298 the key laboratory fund for scientific research in Sichuan (003Z1401). The experiments were 299 conceived and coordinated by HY, YYT, YLL, WY, ZYW ,LZQ and CPY. Sampling was 300 performed by HY, YLL and YYT. Molecular docking was performed by HY, YLL, HL and JZ. 301 HY, YLL and WY drafted the manuscript. All authors read and approved the final version of the 302 manuscript submitted for publication. The authors thank Liwen Bianji, Edanz Editing China 303 (www.liwenbianji.cn/ac), for editing the English text of a draft of this manuscript. 
305

306

307

308

309

310

311

312

313

314

315

316

317

318

319

320

321

322

323

Figure legends

Figure 1 Three-dimensional (3D) model of CbuqPBP1. (A) Sequence alignment between CbuqPBP1 and NribOBP3. (B) 3D structure of CbuqPBP1. The $\mathrm{N}$ and $\mathrm{C}$ termini and the six $\alpha-$ helices are labeled and the three disulfide linkages are shown in yellow stick representations. (C)

Superimposed penetrative structure of CbuqPBP1 and NribOBP3. The model of CbuqPBP1 and crystal structure of NribOBP3 are shown in green and violet, respectively. (D) The results of the PROCHECK evaluation of the CbuqPBP1 model. (E) Overall model quality.

Figure 2 The binding pocket of CbuqPBP1 and the docking result with dibutyl phthalate.

(A) Tertiary structure of dibutyl phthalate. (B) The binding pocket of CbuqPBP1 and dibutyl phthalate docked into the active site of the CbuqPBP1 receptor. (C) Diagram of the van der Waals interactions and hydrophobic interactions of dibutyl phthalate with key binding site residues.

Figure 3 Double digestion map of the mutant and wild-type plasmids. Lane Marker: protein molecular weight standard; Lane 1: pET-28a (+)/PBP1-Phe69A; Lane 2: pET-28a (+)/PBP1His53A; and Lane 3: pET-28a (+)/PBP1.

Figure 4 SDS-PAGE analysis of the total bacterial protein lysate of the mutant and wildtype CbuqPBP1. (A) CbuqPBP1-His53A mutant. Lane 1: IPTG induced total protein lysate; Lane 2: total protein lysate without IPTG induction. (B) CbuqPBP1- Phe69A mutant. Lane 1: total protein lysate without IPTG induction; Lane 2: IPTG induced total protein lysate. (C) wild- 
324 type CbuqPBP1. Lane 1: IPTG induced total protein lysate; Lane 2: total protein lysate without 325 IPTG induction.

326 Figure 5 SDS-PAGE analysis of supernatant and precipitant of bacterial fragmentation 327 following expression of the mutant and wild-type CbuqPBP1. Lane 1: IPTG induced 328 expression of insoluble material; Lane 2: IPTG induced expression of supernatant following cell 329 disruption by sonication. (A) wild-type protein. (B) CbuqPBP1-His53A mutant. (C) CbuqPBP1330 Phe69A mutant.

331

332

Figure 6 The binding curve and $K_{d}$ of mutant and wild-type CbuqPBP1 toward 1-NPN. (A) wild-type protein. (B) CbuqPBP1-His53A mutant. (C) CbuqPBP1- Phe69A mutant.

\section{Figure 7 Competitive binding curves of selected ligands toward mutant and wild-type} CbuqPBP1. (A) wild-type protein. (B) CbuqPBP1-His53A mutant. (C) CbuqPBP1- Phe69A mutant.

\section{Reference}

Cavasotto, C.N., and S.S. Phatak. 2009. Homology modeling in drug discovery: current trends and applications. Drug Discov Today 14: 676-683.

Damberger, F.F., Y. Ishida, W.S. Leal and K. Wuthrich. 2007. Structural basis of ligand binding and release in insect pheromone-binding proteins: NMR structure of Antheraea polyphemus PBP1 at pH 4.5. J Mol Biol 373: 811-819.

Deng, S.S., J. Yin, T. Zhong and Y.Z. Cao. 2011. Function and immunocytochemical localization of two novel odorant-binding proteins in olfactory sensilla of the scarab beetle Holotrichia oblita Faldermann (Coleoptera: Scarabaeidae). Chemical Senses 37: 141-150. 
345

346

347

348

349

350

351

352

353

354

355

356

357

358

359

360

361

362

363

364

365

366

367

368

369

370

371

372

373

Gu, S.H., S.P. Wang, X.Y. Zhang, K.M. Wu, Y.Y. Guo, J.J. Zhou and Y.J. Zhang. 2011. Identification and tissue distribution of odorant binding protein genes in the lucerne plant bug Adelphocoris lineolatus (Goeze). Insect Biochem Mol Biol 41: 254-263.

Huey, R., G.M. Morris, A.J. Olson and D.S. Goodsell. 2007. A semiempirical free energy force field with chargebased desolvation. J Comput Chem 28: 1145-1152.

Jiang, Q.Y., W.X. Wang, Z. Zhang and L. Zhang. 2009. Binding specificity of locust odorant binding protein and its key binding site for initial recognition of alcohols. Insect Biochem Mol Biol 39: 440-447.

Ju, R.T., C.H. Xiao, J.H. Xu, Y. Xu, X.Z. Chi and Y.Z. Li. 2005. Cyrthotrachelus buqueti in Shanghai. Forest Pest and Disease 24: 7-9.

Kozmon, S., R. Matuska, V. Spiwok and J. Koca. 2011. Dispersion interactions of carbohydrates with condensate aromatic moieties: theoretical study on the $\mathrm{CH}-$ pi interaction additive properties. Phys Chem Chem Phys 13 : 14215-14222.

Kruse, S.W., R. Zhao, D.P. Smith and D.N. Jones. 2003. Structure of a specific alcohol-binding site defined by the odorant binding protein LUSH from Drosophila melanogaster. Nat Struct Biol 10: 694-700.

Larsson, M.C., A.I. Domingos, W.D. Jones, M.E. Chiappe, H. Amrein and L.B. Vosshall. 2004. Or83b encodes a broadly expressed odorant receptor essential for Drosophila olfaction. Neuron 43: 703-714.

Li, Y.H., P.Y. Deng, Y.M. Liu and Z.H. Lei. 2016. Binding mechanisms of sex pheromone with pheromone binding and receptor proteins in Plutella xylostella. Journal of Southern Agriculture 47: 928-933.

Mang, D.Z., Q.H. Luo, M. Shu and W. Wei. 2012. Extraction and identification of cuticular semiochemical components of Cyrtotrachelus buqueti Guerin-Meneville (Coleoptera: Curculionidae). Acta Entomol Sin 55: $291-302$.

Mao, Y., X. Xu, W. Xu, Y. Ishida, W.S. Leal, J.B. Ames and J. Clardy. 2010. Crystal and solution structures of an odorant-binding protein from the southern house mosquito complexed with an oviposition pheromone. Proc Natl Acad Sci U S A 107: 19102-19107.

Morris, G.M., R. Huey, W. Lindstrom, M.F. Sanner, R.K. Belew, D.S. Goodsell and A.J. Olson. 2009. AutoDock4 and AutoDockTools4: Automated docking with selective receptor flexibility. J Comput Chem 30: 2785-2791.

Northey, T., H. Venthur, F. De Biasio, F.X. Chauviac, A. Cole, K.A.J. Ribeiro, G. Grossi, P. Falabella, L.M. Field, N.H. Keep and J.J. Zhou. 2016. Crystal Structures and Binding Dynamics of Odorant-Binding Protein 3 from two aphid species Megoura viciae and Nasonovia ribisnigri. Sci Rep 6: 24739.

Sandler, B.H., L. Nikonova, W.S. Leal and J. Clardy. 2000. Sexual attraction in the silkworm moth: structure of the

Peer] reviewing PDF | (2019:04:36358:3:0:NEW 19 Aug 2019) 
374

375

pheromone-binding-protein-bombykol complex. Chem Biol 7: 143-151.

Sun, H., L.X. Zhao, F.R. Zhou and H. Gao. 2013. Pheromone binding protein of Helicoverpa zea based on Homology modeling. Ningxia Journal of Agriculture and Forestry Science and Technology 54: 37-39.

Thode, A.B., S.W. Kruse, J.C. Nix and D.N. Jones. 2008. The role of multiple hydrogen bonding groups in specific alcohol binding site in protein: Insights from structural studies of LUSH. Journal of Molecular Biology 376: 13601376.

Tian, Z.Q., L.N. Sun, Y.Y. Li, H.J. Zhang, W.T. Yan, Q. Yue, L.F. Quan and G.S. Qiu. 2017. Research progress of the function of sex pheromone binding protein in insects. Journal of Agriculture 7: 14-20.

Vogt, R.G., and L.M. Riddiford. 1981. Pheromone binding and inactivation by moth antennae. Nature 293: $161-163$. Wang, W.D., F.Z. Chen and X.Q. Wang. 2005. Reproductive behavior of Cyrtotrachelus buqueti. Sichuan J Zool 24: 540-541.

Wang, Y., H. Sun, J.Y. Zhu, N. Zhao and B. Yang. 2015. Homology modeling of the odorant binding protein of the pine shoot beetle, Tomicus yunnanensis. Chinese Journal of Applied Entomology 54: 1223-1228.

Yang, H., T. Su, W. Yang, C. Yang, L. Lu and Z. Chen. 2017a. The developmental transcriptome of the bamboo snout beetle Cyrtotrachelus buqueti and insights into candidate pheromone-binding proteins. PLoS One 12: e0179807.

Yang, H., T. Su, W. Yang, C.P. Yang, Z.M. Chen, L. Lu, Y.L. Liu and Y.Y. Tao. 2017b. Molecular characterization, expression pattern and ligand-binding properties of the pheromone-binding protein gene from Cyrtotrachelus buqueti. Physiol Entomol 42: 1-10.

Yang, H., T. Su, W. Yang, C.P. Yang, X.L.Zhou and Y. P. Li 2018. Molecular Cloning and Expression Analysis of CbuqPBP1 Gene in the Bamboo Snout Beetle,Cyrtotrachelus buqueti. Journal of Sichuan Agricultural University 36:78-85.

Yang, H., W. Yang, C.P. Yang, Y. Cai, Y.F. Pu, Y.W. Fu and Z.R. He. 2015. Mating behavior of Cyrtotrachelus buqueti (Coleoptera: Curculionidae). Acta Entomol Sin 58: 60-67.

Yang, Y.J., H. Qin, S.F. Wang, Y.P. Wang, H. Liao and S.G. Li. 2010. Antennal ultrastructure and electroantennogram responses of Cyrtotrachelus buqueti Guerin-Meneville (Coleoptera: Curculionidae) to volatiles of bamboo shoot. Acta Entomol Sin 53: 1087-1096.

Yang, Y.J., S.F. Wang, J.W. Gong, C. Liu, C. Mu and H. Qin. 2009. Relationships among Cyrtotrachelus buqueti larval density and wormhole number and bamboo shoot damage degree. Chinese J Appl Ecol 20: 1980-1985.

Ye, Y.X., J.J. Liang, H.P. Wang, Z. Meng, X.D. Zhang and L.X. Xu. 2015. Noncovalent surface functionalization of 
403 multi-walled carbon nanotubes with polyhedral oligomeric silsesquioxane nanoparticles based on $\mathrm{CH}-\pi$ 404 interactions. Acta Polymerica Sinica 4: 427-436.

405 Zhao, C., P. Li, M.D. Smith, P.J. Pellechia and K.D. Shimizu. 2014. Experimental study of the cooperativity of CH- $\pi$ 406 interactions. Org Lett 16: 3520-3523.

407 Zhuang, X., Q. Wang, B. Wang, T. Zhong, Y. Cao, K. Li and J. Yin. 2014. Prediction of the key binding site of odorant408 binding protein of Holotrichia oblita Faldermann (Coleoptera: Scarabaeida). Insect Mol Biol 23: $381-390$.

409 Zhuang, X.J., J. Yin, K.B. Li and Y.Z. Cao. 2013. Bioinformatics analysis of the odorant-binding protein HoblOBP2 in 410 olfactory sesilla of the scarab beetle Holotrichia oblita. Plant Protection 39: 50-55.

411

412 
Table $\mathbf{1}$ (on next page)

Mutagenic primers for CbuqPBP1 
Primers

PBP1-F69A-Fm

PBP1-F69A-Rm

PBP1-H53A-Fm

PBP1-H53A-Rm

PBP1-F

PBP1-R
Sequence

5'-aatgcactattttctgtacagcgaaaaaattcgatttgatgaaag-3'

5'-ctttcatcaaatcgaatttttcgctgtacagaaaatagtgcatt-3'

5'-gatatccaagctctgatgaacgcggaacgaccagtcacccatgc-3'

5'-gcatgggtgactggtcgttccgcgttcatcagagcttggatatc-3'

5'-ggaattccatatgcttagcgaaagcttagttgttgatg-3'

5'-ccgctcgagttaaaaactggtaattccaag-3'

1

2 
Table 2 (on next page)

Binding ability of ligands to mutant and wild-type CbuqPBP1 


\begin{tabular}{|c|c|c|c|c|c|c|}
\hline \multirow{2}{*}{ Ligands } & \multicolumn{3}{|c|}{$\mathrm{IC} 50(\mu \mathrm{M})$} & \multicolumn{3}{|c|}{$\mathrm{Ki}(\mu \mathrm{M})$} \\
\hline & PBP1 & $\mathrm{H} 53 \mathrm{~A}$ & F69A & PBP1 & $\mathrm{H} 53 \mathrm{~A}$ & F69A \\
\hline & & - & & & & \\
\hline benzothiazole & 13.426 & & 10.538 & 9.822 & - & 7.305 \\
\hline dibutyl phthalate & 16.889 & - & 20.04 & 12.355 & - & 13.893 \\
\hline cedrol & 29.953 & - & - & 21.912 & - & - \\
\hline
\end{tabular}

1

2 


\section{Figure 1}

Three-dimensional (3D) model of CbuqPBP1

(A) Sequence alignment between CbuqPBP1 and NribOBP3. (B) 3D structure of CbuqPBP1.

The $\mathrm{N}$ and $\mathrm{C}$ termini and the six a-helices are labeled and the three disulfide linkages are shown in yellow stick representations. (C) Superimposed penetrative structure of CbuqPBP1 and NribOBP3. The model of CbuqPBP1 and crystal structure of NribOBP3 are shown in green and violet, respectively. (D) The results of the PROCHECK evaluation of the CbuqPBP1 model. (E) Overall model quality. 
A

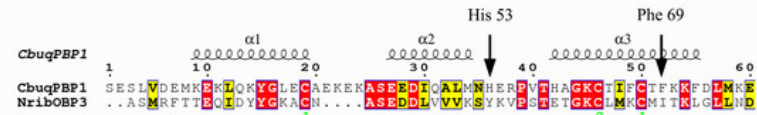

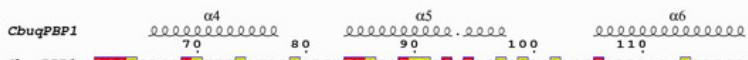

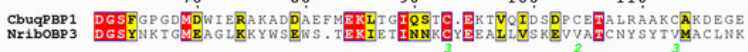

CbuqPBP $12 \frac{e}{120}$

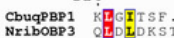

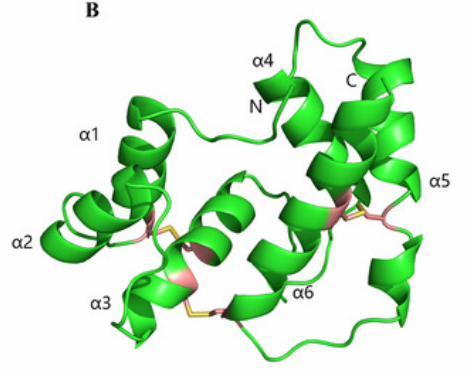

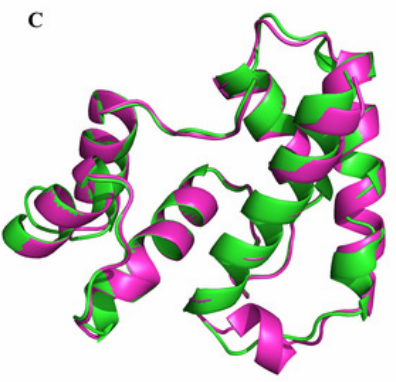

D

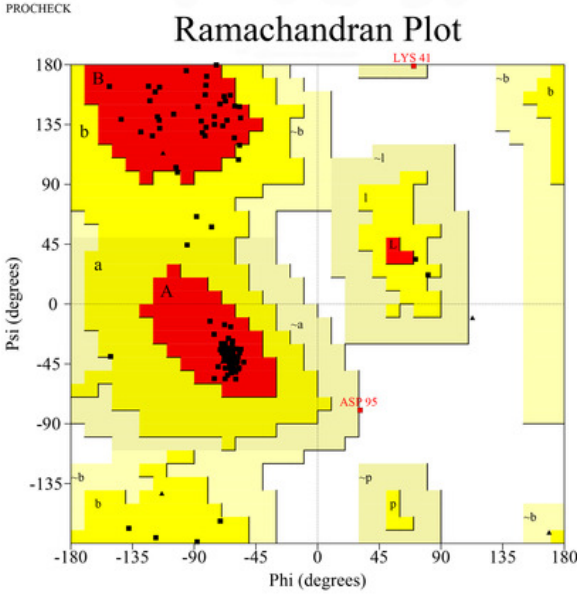

E

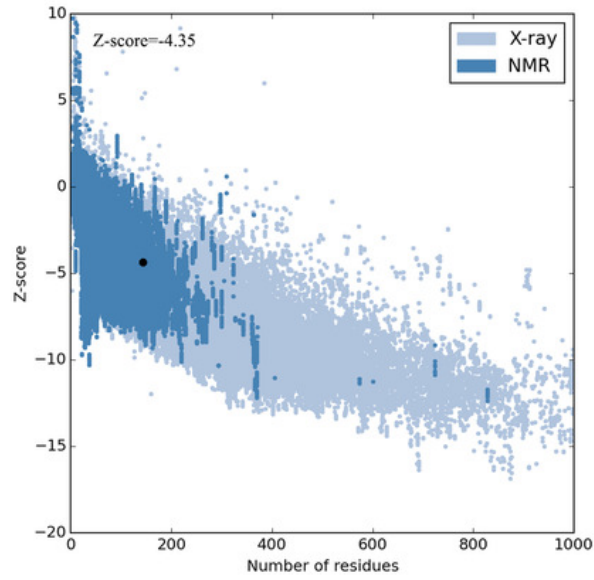


Figure 2

The binding pocket of CbuqPBP1 and the docking result with dibutyl phthalate

(A) Tertiary structure of dibutyl phthalate. (B) The binding pocket of CbuqPBP1 and dibutyl phthalate docked into the active site of the CbuqPBP1 receptor. (C) Diagram of the van der Waals interactions and hydrophobic interactions of dibutyl phthalate with key binding site residues. 
$\mathbf{A}$

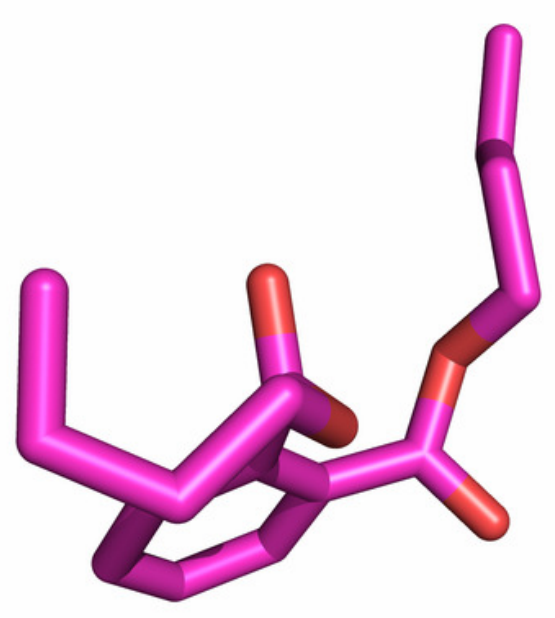

B

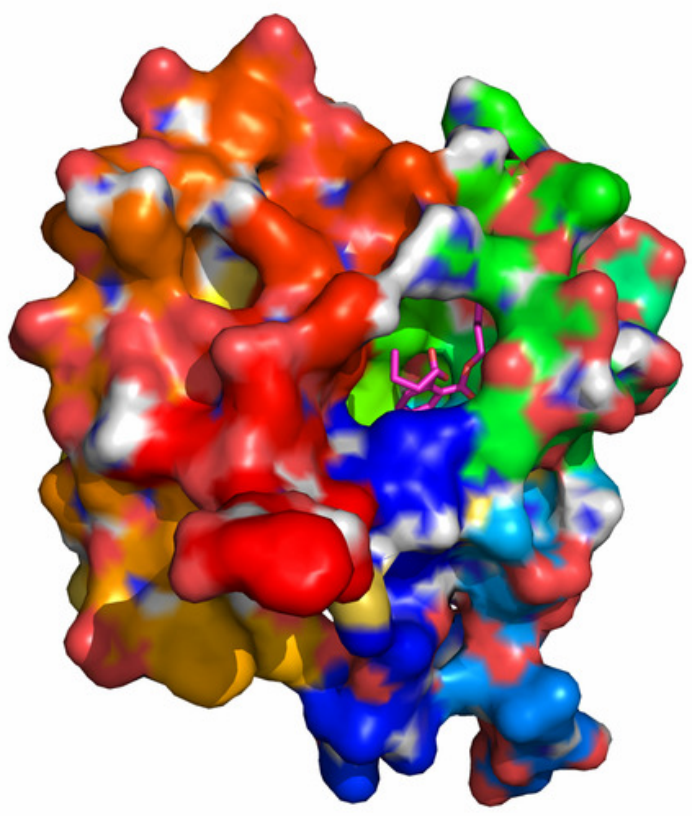

C

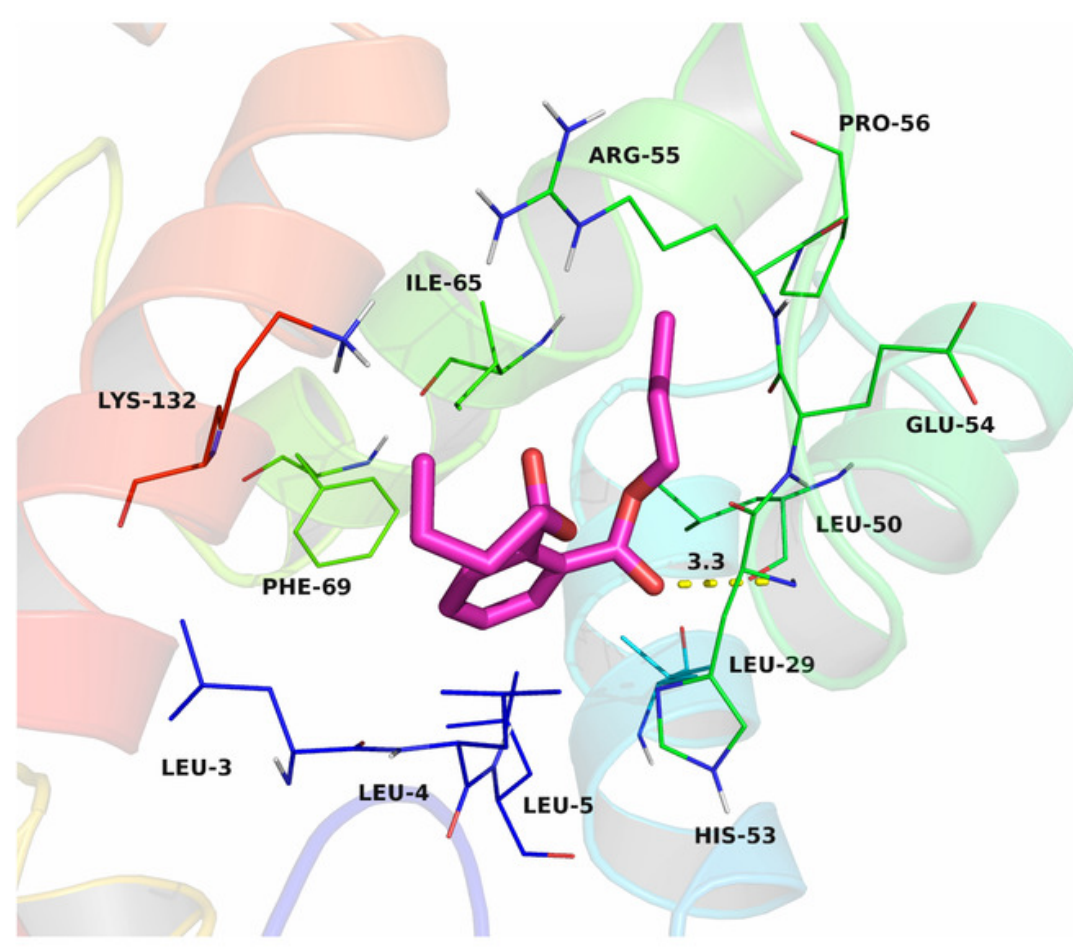


Figure 3

Double digestion map of the mutant and wild-type plasmids

Lane Marker: protein molecular weight standard; Lane 1: pET-28a (+)/PBP1-Phe69A; Lane 2:

pET-28a (+)/PBP1-His53A; and Lane 3: pET-28a (+)/PBP1. 


\section{bp Marker 1123}

\section{0}

1000

750

500

250

100 
Figure 4

SDS-PAGE analysis of the total bacterial protein lysate of the mutant and wild-type CbuqPBP1

(A) CbuqPBP1-His53A mutant. Lane 1: IPTG induced total protein lysate; Lane 2: total protein lysate without IPTG induction. (B) CbuqPBP1- Phe69A mutant. Lane 1: total protein lysate without IPTG induction; Lane 2: IPTG induced total protein lysate. (C) wild-type CbuqPBP1. Lane 1: IPTG induced total protein lysate; Lane 2: total protein lysate without IPTG induction.
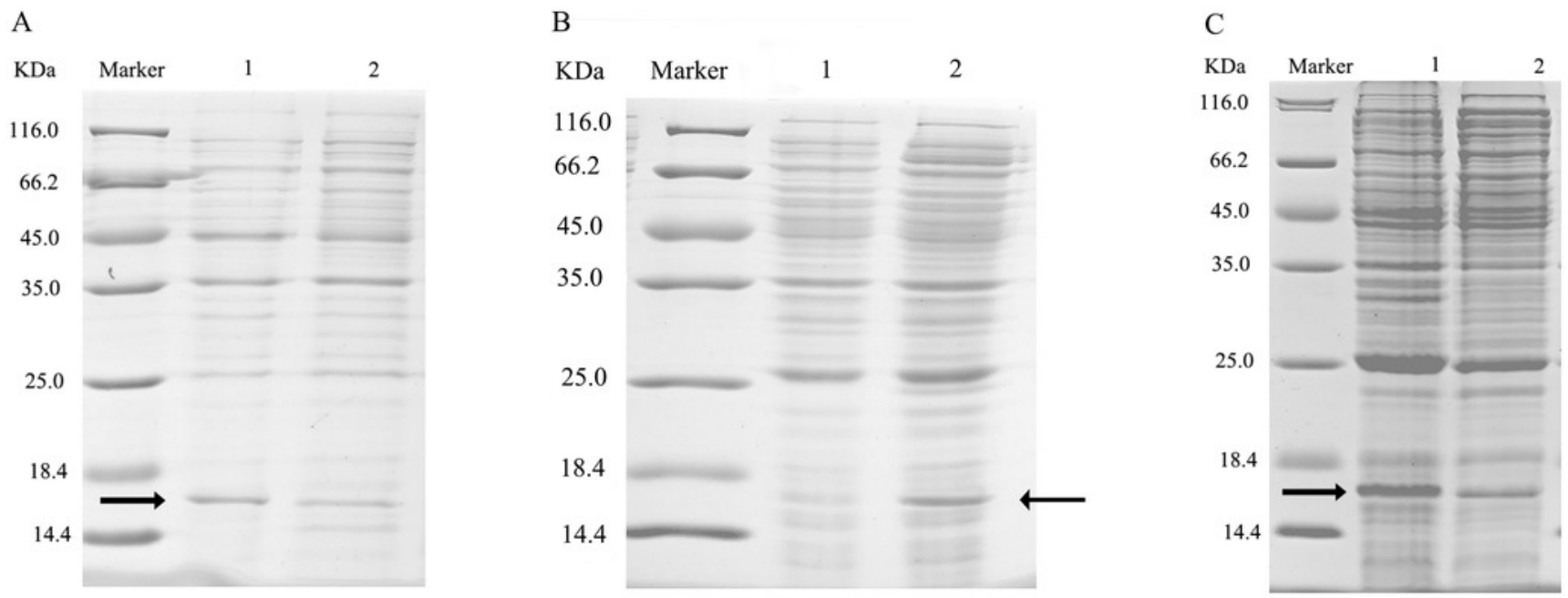
Figure 5

SDS-PAGE analysis of supernatant and precipitant of bacterial fragmentation following expression of the mutant and wild-type CbuqPBP1

Lane 1: IPTG induced expression of insoluble material; Lane 2: IPTG induced expression of supernatant following cell disruption by sonication. (A) wild-type protein. (B) CbuqPBP1His53A mutant. (C) CbuqPBP1- Phe69A mutant.

$\mathbf{A}$

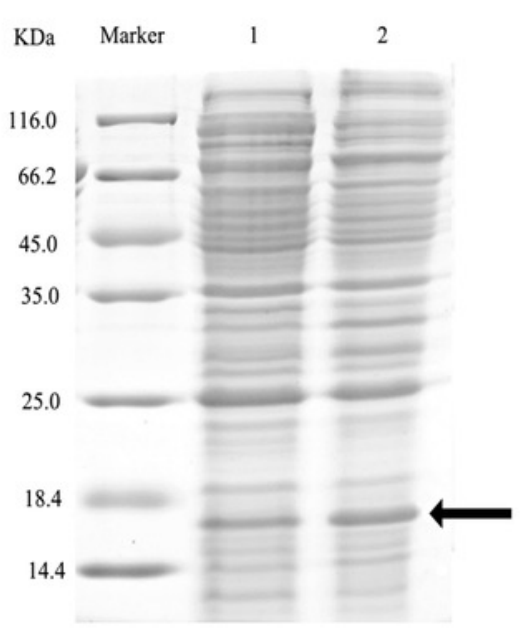

B

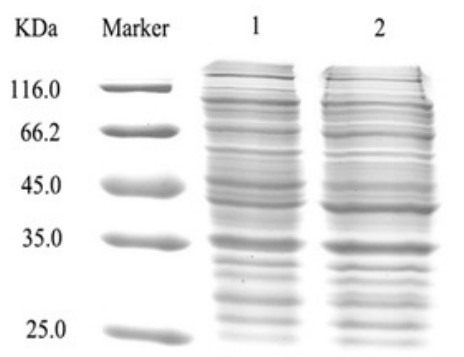

18.4

14.4
C

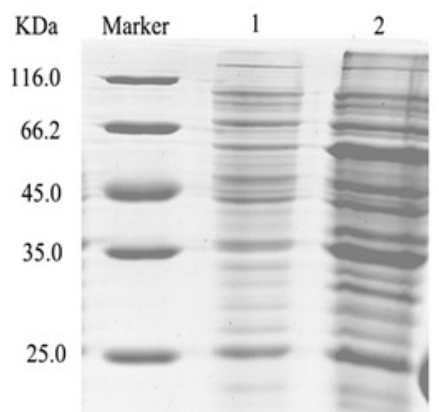


Figure 6

The binding curve and $K_{\mathrm{d}}$ of mutant and wild-type CbuqPBP1 toward 1-NPN

(A) wild-type protein. (B) CbuqPBP1-His53A mutant. (C) CbuqPBP1- Phe69A mutant. 
A

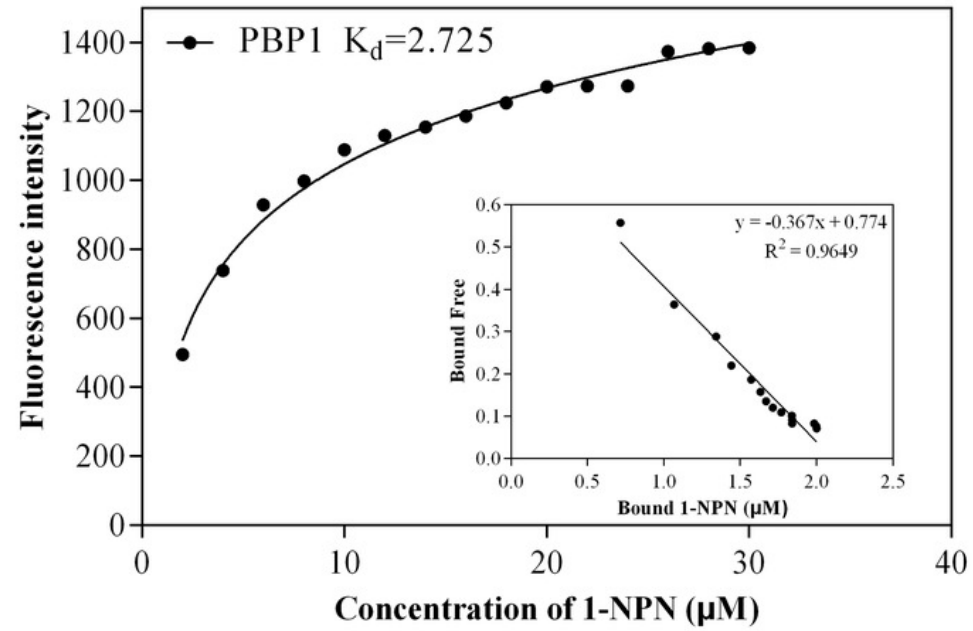

B

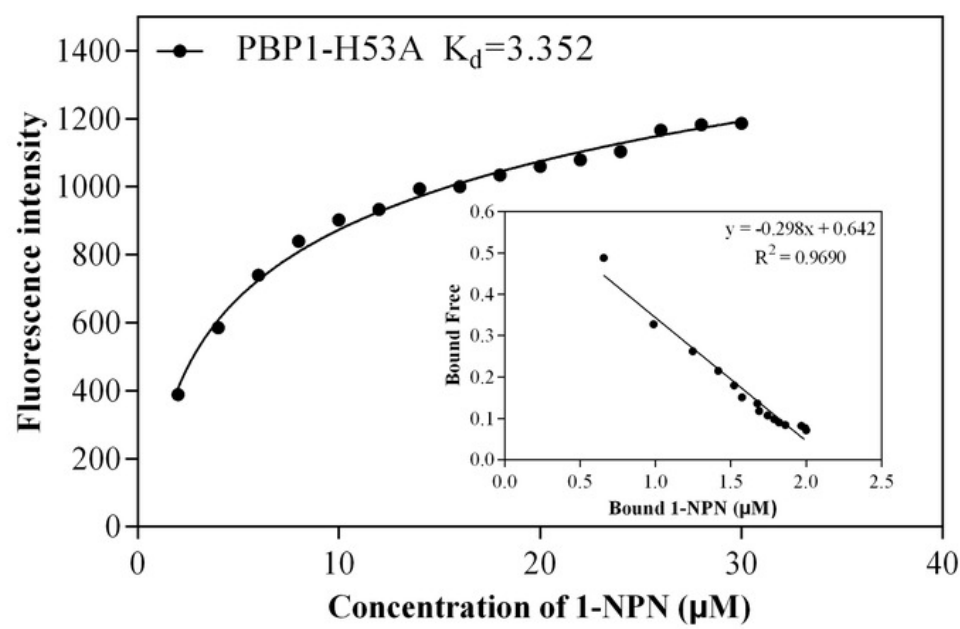

C

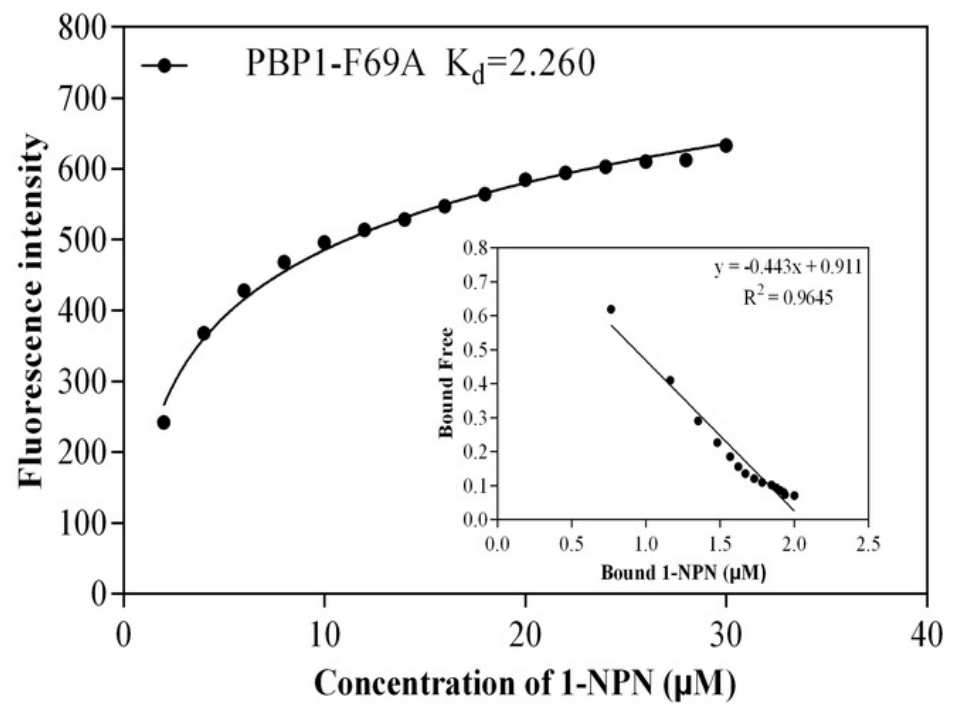


Figure 7

Competitive binding curves of selected ligands toward mutant and wild-type CbuqPBP1
(A) wild-type protein. (B) CbuqPBP1-His53A mutant. (C) CbuqPBP1- Phe69A mutant. 
A

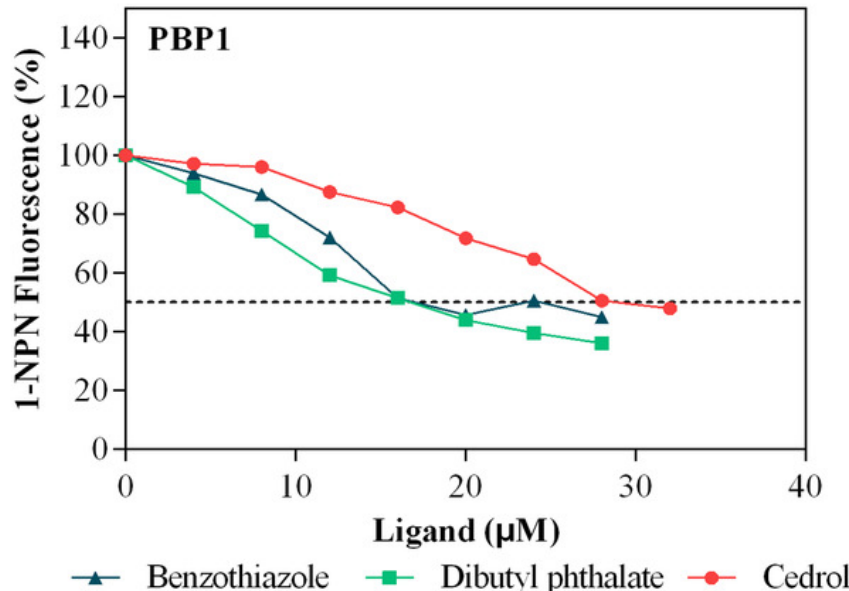

B

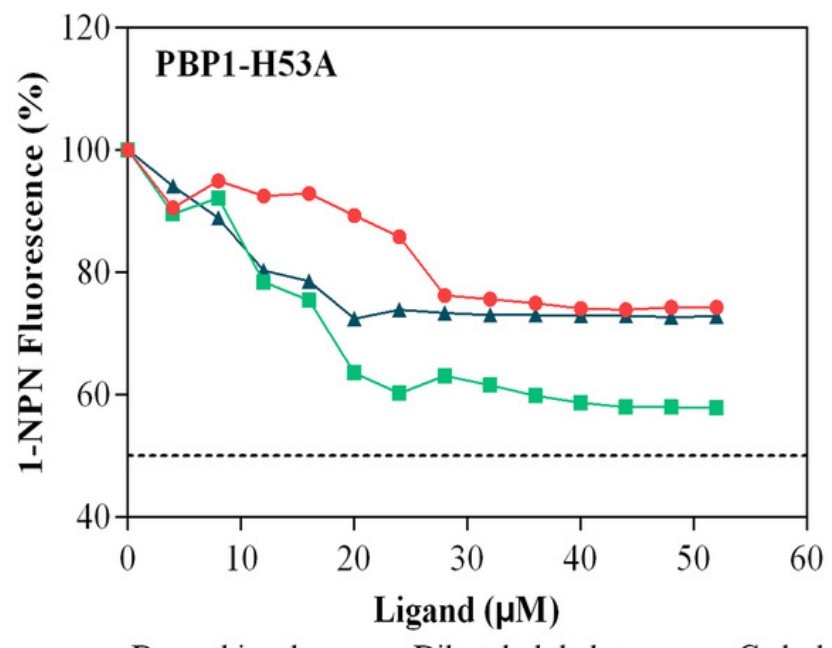

$₫$ Benzothiazole -- Dibutyl phthalate $\rightarrow$ Cedrol

C

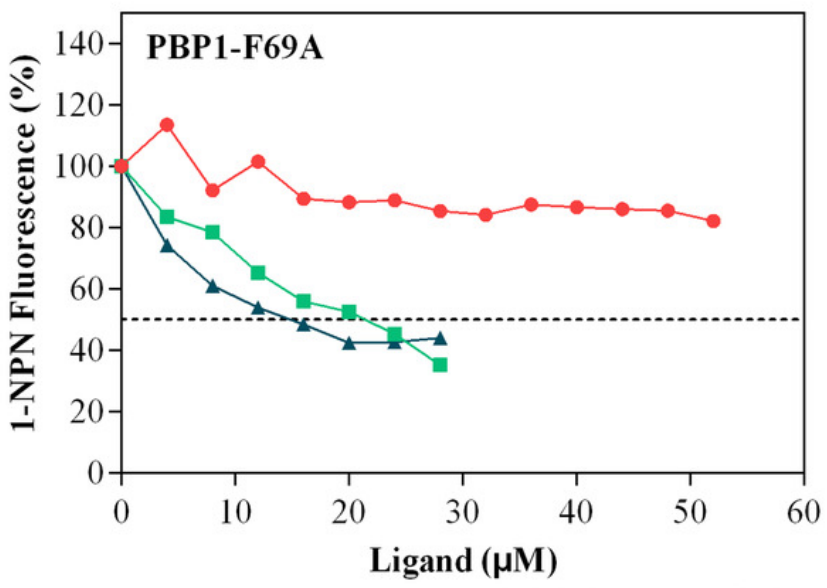

$\leftarrow$ Benzothiazole $\rightarrow$ Dibutyl phthalate $\rightarrow$ Cedrol 